\title{
Innate and Adaptative Immunity in the Sea-Star Asterias Rubens
}

\author{
Michel Leclerc \\ Immunologie des Invertébrés \\ Université d'Orléans 45100, ORLEANS Cedex 2, France
}

Received 2012-06-14, Revised 2012-08-30; Accepted 2012-09-01

\begin{abstract}
The axial organ of the sea star Asterias rubens is a primitive immune organ. The B-like cells, when stimulated by various antigens, produce antibody substances correlating with Mouse Ig Kappa genes. On the other hand elements of the innate immunity are present in Asterias rubens such as Toll-like receptors, interleukin receptors, complement components which contribute to initiate the «antibody factor».
\end{abstract}

Keywords: Asterias Rubens, Innate Immunity, Complement, Primitive Immune, Adaptative Immunity

\section{INTRODUCTION}

A large number of investigations performed in the last few years, in our Laboratory, have provided evidence that the sea star Asterias rubens (echinoderma) posseses a primitive immune system with cellular and humoral responses functionally similar to those of the immune system of vertebrates. It appears that all the elements necessary to these responses are present in the axial organ, which can then be considered as an ancestral lymphoid organ. Two main population of cells are present in this organ: phagocytic cells and cells that are morphologically and functionnaly similar to the lymphocytes of vertebrates.

When isolated axial organ cells were stimulated "in vitro" by a variety of antigens, a soluble factor was secreted in the culture medium, that specifically lysed sheep red cells sensitived with the corresponding antigen (Brillouet et al., 1984). This lytic reaction required the presence of thermolabile factor (complement) present in mammal serum . Moreover, it could be demonstrated that this antibody factor was produced by the B-like cell but only when T-like and phagocytic cells were present during the antigenic stimulation. A detail review of these findings has already been published. Brillouet et al. (1984) and Leclerc et al. (1986) we have observed that the antibody factor showed some homologies with human kappa-like (Leclerc, 2000) and correlated with Mouse Ig kappa precursor genes (Leclerc et al., 2011).

\subsection{Innate Immunity}

We have also observed that the sea-star A.rubens present a panel of Toll-like receptor genes. When compared to other Invertebrates and because the immune function of Toll in Drosophila was not then known...It was assumed that TIL (now known as TLR1) might participate in mammalian development. However, in 1991, it was observed that a molecule, with a clear role, in immune function in mammals: the interleukin-1 (IL-1) receptor (found also in sea-star), had homology to drosophila Toll, the cytoplasm portions of both molecules were similar (Gay and Keith, 1991) always in drosophila, in 1997 TLR4, as an LPS sensing receptor was discovered. TLR4 plays a role for initiating an adaptative immune response (Medzhitov et al., 1997). On the other hand, Toll-like receptor genes have been recently discovered in the sea-urchin (Rast et al., 2006) In the present study, we have analysed the Toll-like receptor genes of the immunized sea-star Asterias rubens and the control ones.

\section{MATERIALS AND METHODS}

Immunization was performed on 20 sea-stars, in an aquarium with sea water at $10^{\circ} \mathrm{C}$ by using Horse-Radish Peroxydase (HRP) (Sigma Products) as antigen at a concentration of $1 \mathrm{mg} \mathrm{mL} \mathrm{m}^{-1}$. 20 non -injected animals were used as controls. The axial organs were removed; RNA was extracted, using Trizol (Invitrogen) according 
to manufacturer instructions, from immunized sea stars (HRP) and Controls (C). cDNA was normalized using double strand specific nuclease essentially as described by Zhulidov et al. (2004). cDNA was fragmented using DNA Fragmentase (New England Biolabs), according to the manufacturer's instructions. After ligation of adapters for illumina's GSII sequencing system, the cDNA was sequenced on the illumina GSII platform sequencing $1 \times 100 \mathrm{bp}$ from one side of the approximately $200 \mathrm{bp}$ fragments. Sequences were assembled using Velvet (Zerbino and Birney (2008).

\section{RESULTS}

First results concern significative Toll-like receptor genes found in immunized sea-starsI) HRP (Immunized animal): TLR13_MOUSE Toll-like receptor 13 and TLR 12 MOUSE TLR-Like receptor (homologies of $63 \%$ with mouse).

Second results concern Toll-like receptors found in control sea-stars (non-immunized):

- TLR1 Toll-like receptor1

- $\quad$ TLR4 Toll-like receptor 4

- TLR3_Toll-like receptor 3

- TLR5 Toll-like receptor 5

- $\quad$ TLR7 Toll-like receptor 7

- $\quad$ TLR9 Toll-like receptor 9

Genes playing a key role in the innate immune system as TLR1-TIL are present in A.rubens and Strongylocentrotus purpuratus (sea-urchin). TLR4 was also found in Drosophila (Medzhitov et al., 1997), A.rubens and, TLR3, TLR5, TLR7 and TLR9. We must notice that only the sea-star (among Invertebrates) presents TLR12 and TLR13 genes, after immunization. We have to say that the sea-star immune model remains enigmatic in terms of immunization.

Finally, an effective innate immune system is already present in Echinoderma as shown in sea-urchin (Hibino et al., 2006) and sea-star and in Invertebrates like in Insects.

\subsection{Innate Immunity}

Cytokins Non-adherent-nylon wool cells(T-like subpopulation) can release soluble lymphokine-like mediators, after stimulation with P.W.M sepharose $6 \mathrm{MB}$ beads, with mitogenic properties (Leclerc et al., 1984). Some interleukins were discovered in Legac et al. (1996) such as IL1.

\subsection{Results}

We describe first «interleukin genes» then « interleukin receptor genes » as compared to mammals.

\subsection{Control-« Nuclear Factor Interleukin-3- Regulated Protein »}

The results of our BlastX (Blast Version 2.2.20, Parameters:-e 0.001 -F F -b 3 -v 3 -I T -a 16 -m 7) were the following. One contig (NODE_3558_length_451_cov_24.356985) could be annotated via BLASTX to mouse "Nuclear factor interleukin-3-regulated protein" from the SWISSPROT database, with an e-value of $2.87689 \mathrm{e}-10$. On an aligned region of 62 amino acids, 42 positive and 28 identical amino acids were found.

\subsection{HRP-« Nuclear Factor Interleukin-3- Regulated Protein »}

One contig (NODE_48893_length_689_cov_15.156749) could be annotated via BLASTX to mouse "Nuclear factor interleukin-3-regulated protein" from the SWISSPROT database, with an e-value of $1.49652 \mathrm{e}-09$. On an aligned region of 57 amino acids, 40 positive and 26 identical amino acids were found. We study now the interleukin 1 and more specially interleukin 1 receptors:

\subsection{Control-«X-Linked Interleukin-1 Receptor Accessory Protein-Like2 »}

One

contig (NODE_21299_length_1163_cov_23.764402) could be annotated via BLASTX to mouse "X-linked interleukin1 receptor accessory protein-like 2" from the SWISSPROT database, with an e-value of 2.64154e-07. On an aligned region of 278 amino acids, 110 positive and 60 identical amino acids were found. (b) HRP-«Xlinked interleukin-1 receptor accessory protein-like $2 »$ : One contig (NODE_23687_length_300_cov_7.280000) could be annotated via BLASTX to mouse "X-linked interleukin-1 receptor accessory protein-like 2" from the SWISSPROT database, with an e-value of 7.2295e-05. On an aligned region of 77 amino acids, 38 positive and 27 identical amino acids were found. And now the interleukin-1 receptor-associated kinase 4 :

\subsection{Control-« Interleukin-1 Receptor- Associated Kinase 4 » \\ One contig (NODE_45921_length_271_cov_7.907749) could be}


annotated via BLASTX to mouse "Interleukin-1 receptor-associated kinase 4" from the SWISSPROT database, with an e-value of 0.000100449 . On an aligned region of 96 amino acids, 37 positive and 22 identical amino acids were found.

\subsection{HRP-« Interleukin-1 Receptor-Associated Kinase 4 »}

No contig matched. We finish with the interleukin17 receptor $\mathrm{B}$ as compared to mammals:

\subsection{Control-«Interleukin-17 Receptor B »}

One

contig

(NODE 31878 length 179 cov 15.167598) could be annotated via BLASTX to mouse "Interleukin-17 receptor B" from the SWISSPROT database, with an e-value of 0.000617054 . On an aligned region of 42 amino acids, 24 positive and 14 identical amino acids were found.

\subsection{HRP-« Interleukin-17 Receptor B »}

One

contig (NODE_46091_length_308_cov_16.399351) could be annotated via BLASTX-mouse "Interleukin-17 receptor B" from the SWISSPROT database, with an e-value of 0.000279731 . On an aligned region of 49 amino acids, 28 positive and 16 identical amino acids were found. As shown, it exists also an Interleukin-17 receptor A:

\subsection{Control-« Interleukin-17 Receptor A»}

No contig matched.

\subsection{HRP-« Interleukin-17 Receptor A »}

One

contig NODE_13602_length_446_cov_23.168161) could be annotated via BLASTX to mouse "Interleukin-17 receptor A" from the SWISSPROT database, with an e-value of 0.000671577 . On an aligned region of 81 amino acids, 38 positive and 24 identical amino acids were found. The immunization leads to modifications in the case of «Interleukin-1 receptor-associated kinase 4 » and in the case of «Interleukin-17 receptor A » where this last appears in immunized animals.Furthermore interleukin genes(most of them corresponding to interleukin receptor genes) such as IRPL2, IRAK 4, IL-17RB, IL-17RA are present in the sea-star Asterias rubens.

How to explain this phenomenon? To try to interprate it could be a challenge as compared to mammal interleukins. Nevertheless these observations would indicate that certain interleukins and particularly
« receptors »recalling mammal ones are present in the immune system of the sea star.

We note specially, in mammals that, the interleukin 17 receptor $\mathrm{B}$ is implicated in the immune response by mediating the activation of NF-Kappa B; that the interleukin 17 receptor A belongs to a novel family of inflammatory cytokines. As for the IRAK 4, it is required for various responses induced by Il-1R and toll-like receptor signals Innate Immunity: complement components.

In earlier results, complement-like activity, was found in another sea-star: Asterias forbesi (Leonard et al., 1990). As for Asterias rubens, observed in it genome, we can say: At first, Components of the classical pathway as compared to mammals, were discovered.

\subsection{Control-Complement C1q Subcomponent Subunit A}

One contig (NODE_55223_length_184_cov_8.456522) could be annotated via BLASTX to mouse "Complement C1q subcomponent subunit A" from the SWISSPROT database, with an e-value of $8.55742 \mathrm{e}-06$. On an aligned region of 72 amino acids, 29 positive and 25 identical amino acids were found.

\subsection{HRP-Complement C1q subcomponent subunit A}

No contig matched, no hit was found. Then,

\subsection{Control-Complement C1q Subcomponent Subunit B}

One

contig (NODE_48557_length_161_cov_19.316771) could be annotated via BLASTX to mouse "Complement C1q subcomponent subunit B" from the SWISSPROT database, with an e-value of 8.55742e-06. On an aligned region of 57 amino acids, 33 positive and 19 identical amino acids were found.

\subsection{HRP-Complement C1q Subcomponent Subunit B}

One contig (NODE_68235_length_178_cov_7.724719) could be annotated via BLASTX to mouse "Complement C1q subcomponent subunit B" from the SWISSPROT database, with an e-value of 3.97232e-10. On an aligned region of 44 amino acids, 39 positive and 28 identical amino acids were found Then. 


\subsection{Control-Complement C1q Subcomponent Subunit C}

One

contig

(NODE_37343_length_278_cov_12.356115). On an aligned region of 39 amino acids, 25 positive and 19 identical amino acids were found.

\subsection{HRP-Complement C1q Subcomponent Subunit C}

Three contigs could be annotated via BLASTX to mouse "Complement C1q subcomponent subunit C" from the SWISSPROT database. The best contig (NODE_29181_length_277_cov_7.790614) with an e-

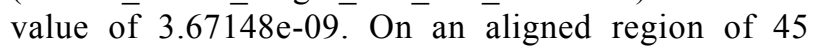
amino acids, 27 positive and 22 identical amino acids were found.

\subsection{We Observe now the C2 Component}

Five contigs could be annotated via BLASTX to mouse "Complement C2" from the SWISSPROT database. The best contig (NODE_23089_length_519_cov_15.136802) with an evalue of $7.65302 \mathrm{e}-07$. On an aligned region of 153 amino acids, 59 positive and 38 identical amino acids were found. And now what about the component C4?

\subsection{Control-Complement C4-B}

One contig (NODE_95392_length_215_cov_10.818604) could be annotated via BLASTX to mouse "Complement C4-B" from the SWISSPROT database, with an e-value of 3.48532e-06. On an aligned region of 76 amino acids, 40 positive and 21 identical amino acids were found.

\subsection{HRP-Complement C4-B}

One contig (NODE_58696_length_164_cov_11.884147) could be annotated via BLASTX to mouse "Complement C4-B" from the SWISSPROT database, with an e-value of $7.51259 \mathrm{e}-05$. On an aligned region of 44 amino acids, 26 positive and 21 identical amino acids were found. Then, we see the $\mathrm{C} 3$ component which is central, in mammals, to both the classical and alternative pathways.

\subsection{Control-Complement C3}

Seven contigs could be annotated via BLASTX to mouse "Complement C3" from the SWISSPROT database. The best contig
(NODE_25262_length_1200_cov_21.958334) with an e-value of $3.2395 \mathrm{e}-49$. On an aligned region of 355 amino acids, 128 positive and 173 identical amino acids were found.

\subsection{HRP-Complement C3}

Eleven contigs could be annotated via BLASTX to mouse "Complement C3" from the SWISSPROT database. The best contig (NODE_15219_length_398_cov_19.402010) with an evalue of $4.96748 \mathrm{e}-24$. On an aligned region of 146 amino acids, 79 positive and 59 identical amino acids were found.It must be noted that $\mathrm{C} 3$ belongs to, also, the ALTERNATE pathway. We give now the obtained results concerning the components of the membraneattack complex as compared to mammals. We begin with the very important C9:

\subsection{Control-Complement Component C9}

Three contigs could be annotated via BLASTX to mouse "Complement component C9" from the SWISSPROT database. The best contig (NODE_46472_length_143_cov_10.104895) with an e-value of $1.41746 \mathrm{e}-0 \overline{5}$. On an aligned region of 31 amino acids, 17 positive and 15 identical amino acids were found.

\subsection{HRP-Complement Component C9}

No contig matched, no hit was found. Then we look about the C5 component:

\subsection{Control-Complement C5}

One contig (NODE_75605_length_236_cov_32.677967) could be annotated via BLASTX to mouse "Complement C5" from the SWISSPROT database, with an e-value of 0.000634854 . On an aligned region of 55 amino acids, 27 positive and 19 identical amino acids were found.

\subsection{HRP-Complement C5}

Two contigs could be annotated via BLASTX to mouse "Complement C5" from the SWISSPROT database. The best contig (NODE_7717_length_665_cov_13.831579) with an evalue of $6.42528 \mathrm{e}-17$. On an aligned region of 212 amino acids, 99 positive and 57 identical amino acids were found. At last we observe the C8 Component. 


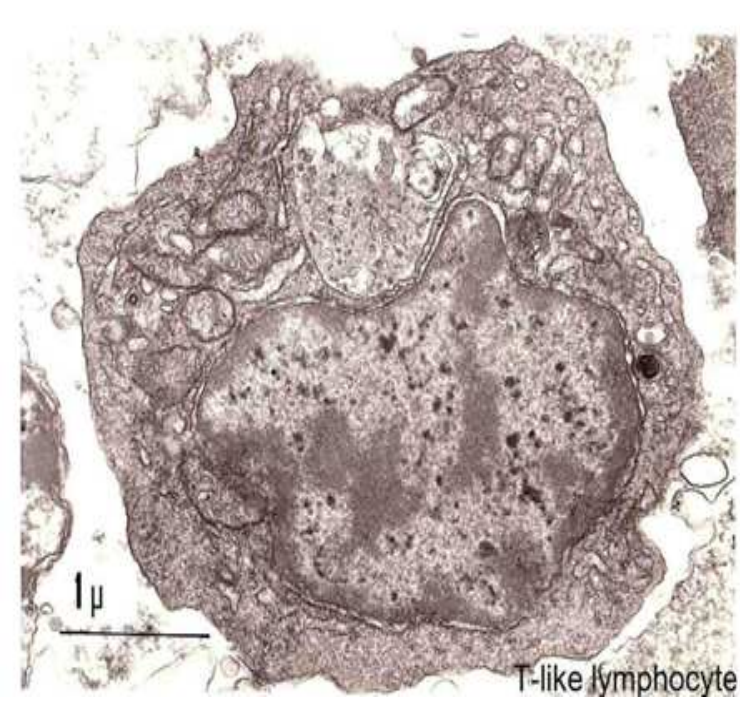

Fig. 1. T-like lymphocyte in T.E.M

\subsection{Control-Complement Component C8 Alpha Chain}

Twelve contigs could be annotated via BLASTX to mouse "Complement component C8 alpha chain" from the SWISSPROT database. The best contig (NODE_68667_length_311_cov_10.845659) with an evalue of $6.7995 \mathrm{e}-15$. On an aligned region of 95 amino acids, 33 positive and 42 identical amino acids were found.

\subsection{HRP-Complement COMPONENT C8 ALPHA CHAIN}

Six contigs could be annotated via BLASTX to mouse "Complement component C8 alpha chain" from the SWISSPROT database. The best contig (NODE_43169_length_2163_cov_11.209893) with an evalue of $6.360 \overline{4} 6 \mathrm{e}-14$. On an aligned region of 45 amino acids, 42 positive and 33 identical amino acids were found.

The main components of Complement as compared to mammal ones were found in the sea star: How genes are expressed? By which pathway (direct or alternative pathway)? It could be a working hypothesis which needs to be explored. However,it is said that in immunized animals, no hits were found with C9 and $\mathrm{Clq}$ (subcomponent subunit A): it remains enigmatic.It could be expected that the sea-star specific immune reaction needs all these genes to be expressed.

\subsection{Adaptative Immunity: «The Antibody Factor»)}

Two main populations of cells are present in the axial organ: phagocytic cells and cells that are morphologically and functionnaly similar to the lymphocytes of vertebrates (Fig. 1). In cooperation with the sea-star complement, they initiate the "antibody factor" (Delmotte et al., 1986) recently kappa genes were discovered in the sea-star A. Rubens (Leclerc et al., 2011). We now present the DNA structure of these genes in immunized and control sea-stars.

We recall that antibody factor was isolated and purified (Delmotte et al., 1986). In 2000, it was shown that it presented homologies with human kappa-like (Leclerc, 2000). Using the same approach (Leclerc et al., 2011), the sea-star genomes (Asterias rubens, Patiria miniata $^{\circ}$ ) were analysed.

\subsection{In Asterias Rubens}

One contig (Immunized sea-stars) (NODE 13406 length $132 \mathrm{cov} 46.075756$ ) could be annonated via BLASTX to mouse "e kappa chain V-III region MOPC 63" from the SWISSPROT database, with an e-value of 0,000458269 . On an aligned region of 39 amino acids, 21 positive and 16 identical amino acids were found. Otherwise, it was shown that: One contig (controls) (NODE_1857_length_169_cov_25.875740) could be annotated via BLASTX-mouse "IG KAPPA CHAIN V-V REGION MOPC 41" from the SWISSPROT database, with an e-value of 0.000886447 . On an aligned region of 33 amino acids, 17 positive and 11 identical amino acids were found. In Patiria miniata ${ }^{\circ}$ also, Ig kappa chain V.III region MOPC 63, Ig kappa chain region MOPC 41 and Ig kappa chain $\mathrm{V}-\mathrm{V}$ region $\mathrm{K} 2$ were detected in a significant manner.

\section{DISCUSSION}

These observations confirm that certain mammal structures are present in the immune system of the sea star and this finding strongly supports the idea that an effective immune system is already present in the seastar: A. rubens. The main acquisition of Echinoderms seems to be the cellular differentiation in two subpopulations of cells, ancestral to $T$ and $B$ lymphocytes and their interplay with phagocytes resulting in the synthesis of specific humoral antibody factor. Although all efforts to find a primitive form of immunoglobulin in sea-urchin genome (Rast et al., 2006) have been unsuccessful, it is the first time, we present arguments indicating that vertebrate immunoglobulin Kappa genes are present in an invertebrate and Deuterostomes. It appears that the antibody factor has a 
molecular weight of 120-130.000 daltons (Delmotte et al., 1986) with 4 sub-units of 30.000 daltons each: it could be composed of 4 « kappa chains ».

\section{CONCLUSION}

In conclusion the "Avenir" of the immune sea-star study is a bright one.

\section{REFERENCES}

Brillouet, C., M. Leclerc and G. Luquet, 1984. PAS-1, a protein affinity purified from Ascaris suum worms, maintains the ability to modulate the immune response to a bystander antigen. Cell. Immunol., 84: 138-144. DOI: 10.1111/j.1440-1711.2005.01404.x

Delmotte, F., C. Brillouet, M. Leclerc, G. Luquet and JC. Kader, 1986. Purification of an antibody-like protein from the sea star Asterias rubens (L.). Eur. J. Immunol., 16: 1325-1330. PMID: 3096739

Gay, N.J. and F.J. Keith, 1991. Drosophila Toll and IL-1 receptor. Nature, 351: 355-356. PMID: 1851964

Hibino, T., M. Loza-Coll and C. Messier, A.J. Majeske and A.H. Cohen, 2006. The immune gene repertoire encoded in the purple sea urchin genome. Dev. Biol., 300: 349-365. PMID: 17027739

Leclerc, M., 2000. Human kappa-like expression in the axial organ of the sea star Asterias rubens (Echinoderma). Eur. J. Morph, 38: 206-207. PMID: 10916174

Leclerc, M., C. Brillouet, G. Luquet and R.A. Binaghi, 1986. The immune system of invertebrates: the sea star Asterias rubens (Echinoderma) as a model of study. Bull. Inst. Pasteur, 84: 311-330.

Leclerc, M., C. Brillouet, G. Luquet, P. Agogue and R.A. Binaghi, 1984. Properties of Cell Subpopulations of Starfish Axial Organ: In Vitro Effect of Pokeweed Mitogen and Evidence of Lymphokine-like Substances. Scand. J. Immunol., 14: 281-284. DOI: 10.1111/j.1365-3083.1981.tb00565.x
Leclerc, M., S. Dupont, O. Ortega-Martinez, B. Hernroth and B. Krezdorn, 2011. Evidence of Kappa genes in the sea-star Asterias rubens (Echinoderma). Immunol. Lett., 138: 197-198. DOI: 10.1016/j.imlet.2011.01.016

Legac, E., G.L. Vaugier, F. Bousquet, M. Bajelan and M. Leclerc, 1996. Primitive cytokines and cytokine receptors in invertebrates: The sea star asterias rubens as a model of study. Scand. J. Immunol., 44: 375-380. DOI: 10.1046/j.1365-3083.1996.d01-322.x

Leonard, L.A., J.D. Strandberg and J.A. Winkelstein, 1990. Complement-like activity in the sea star, Asterias forbesi. Dev. Comp. Immunol., 14: 19- 30. PMID: 2338154

Medzhitov, R., P. Preston-Hurlburt and C.A. Janeway, 1997. A human homologue of the Drosophila Toll protein signals activation of adaptive immunity. Nature, 388: 394-397. PMID: 9237759

Rast, J.P., L.C. Smith and M. Loza-Coll, T. Hibino and G.W. Litman, 2006. Genomic insights into the immune system of the sea urchin. Science, 314: 952956. PMID: 17095692

Zerbino, D.R. and E. Birney, 2008. Velvet: algorithms for de novo short read assembly using de Bruijn graphs. Genome Res., 18: 821-829. DOI: 10.1101/gr.074492.107

Zhulidov, P.A., E.A. Bogdanova, A.S. Shcheglov, L.L. Vagner and G.L. Khaspekov et al., 2004. Simple cDNA normalization using kamchatka crab duplexspecific nuclease. Nucl. Acids Res., 32: e37-e37. PMID: 14973331 